Tohoku J. exp. Med., 1981, 133, 285-288

\title{
The Interference Effect of Simultaneous Vocalization on Manual Reaction Time
}

\author{
Ryuichi Nakamura, Atsushi Isagoda and Tomiyoshi \\ CHIDA \\ Department of Rehabilitation Medicine, Institute of Balneology, \\ Tohoku University School of Medicine, Sendai 980
}

\begin{abstract}
Nakamura, R., Isagoda, A. and Chida, T. The Interference Effect of Simultaneous Vocalization on Manual Reaction Time. Tohoku J. exp. Med., 1981, 133 (3), 285-288 — Simple reaction times (RTs) of the left or right hand with or without simultaneous vocalization were examined in 14 righthanded subjects. RTs of the simultaneous tasks were longer than those of the single tasks and slowing of RT was larger on the manual than vocal response, particularly on the right side. The results are interpreted as reflecting a different mode of processing in the left and right hemispheres.—reaction time; interference effect; hemispheric differentiation
\end{abstract}

Recent studies reported that the manual performance could be varied with the simultaneous speaking. The performance with speaking was less effective than that without speaking, especially on the right hand (Kinsbourne and Cook 1971; Hicks 1975). This interference would be due to a functional overlap of speaking and motor control in the left hemisphere (Lomas and Kimura 1976). Several manual tasks such as dowel balancing (Kinsbourne and Cook 1971; Lomas and Kimura 1976), finger tapping or button-pressing (McFarland and Ashton 1975, 1978; Bowers et al. 1978) and simple reaction time (RT) (Low and Rebert 1978; Rizzolatti et al. 1979) were examined with concurrent mental or verbal performance to analyse the interference effect. Bilateral simultaneous movements took more time to produce motor effect than the separated movement of either arm alone, but the slowing of RT in the simultaneous movement was not different in the left and the right arm (Taniguchi et al. 1977), and the degree of interference was the same in the left and right hand (Nakamura et al. 1977). In the present study, we investigated the simple RT of the left or right hand with simultaneous vocalization, and analysed a possible hemispheric differentiation of the interference effect of vocalization on motor performance.

\section{Method}

The experiments were performed on 14 right-handed laboratory staffs, seven male and seven female, aged from 21 to 43 years.

In a quiet room the subject was seated comfortably on a chair with eyes closed and the forearms rested on a desk. The subject was first given a warning verbal signal

Received for publication, March 6, 1980. 
and 2-4 sec afterwards a peep sound (1000 Hz, $100 \mathrm{~dB}, 50$ msec duration). The subject was beforehand instructed to respond as quickly as possible to the peep sound with extension of the middle finger and/or vocalization, ' $\mathrm{Pa}$ '.

A small metal knob was taped on the ventral surface of the middle finger and made contact with the metal plate on which the hand rested. A switch formed between the metal knob and the plate was opened when the finger lifted, thus signalling the precise instance of 'off time'. About $10 \mathrm{~cm}$ apart from the subject's mouth was placed a unidirectional microphone (AIWA DM-508) which detected the subject's vocal response. Both the off signal and vocal responses were displayed on a memoscope (SANEI 7T07A). The intervals between the stimulus onset and each of the mechanical and vocal responses were measured with a msec scale.

The tasks were as follows: 1) single tasks (three tasks); extension of the left middle finger, that of the right, or vocalization, and 2) simultaneous tasks (two tasks); extension of the left middle finger with vocalization, or extension of the right middle finger with vocalization. These five tasks were tested in a randomized order with each block of five same trials. When the subject failed to respond to a stimulus, the trial was repeated. The interval between trials was 5-10 sec. The geometric means were calculated for 20 trials in each task.

\section{Results}

Table 1 shows the means and standard deviations of RTs in all subjects. RTs of each hand were longer on the simultaneous tasks than on the single tasks. The differences of RTs between the single and simultaneous tasks were calculated in each subject, subtracting RT of a single task from that of a simultaneous task (left hand; $9.6 \mathrm{msec}, t=3.768, \mathrm{~d} f=13, p<0.01$ : right hand; $19.1 \mathrm{msec}, t=6.655$, $\mathrm{d} f=13, p<0.001)$. The differences of RTs between the two tasks were larger on the right hand than on the left hand $(t=5.030, \mathrm{~d} f=13, p<0.001)$. Slowing of vocal response was significant on the simultaneous task with the right hand (difference; $6.8 \mathrm{msec}, t=2.682 \mathrm{~d} f=13, p<0.05$ ), but not with the left hand (difference; $0.8 \mathrm{msec}, t=0.240, \mathrm{~d} f=13$ ). In the simultaneous task of the right manual and vocal responses compared with those in the single task, the extent of slowing was larger on the manual than vocal response $(t=3.982, \mathrm{~d} f=13, p<0.01)$, whereas there appeared to be a greater slowing of the left manual than vocal response, though not significant $(t=2.007, \mathrm{~d} f=13, p<0.1)$.

TABLE 1. Means and standard deviations (in msec) of RTs

\begin{tabular}{lrrrrrrrrr}
\hline & \multicolumn{3}{c}{ Single task } & & \multicolumn{4}{c}{ Simultaneous task } \\
\cline { 2 - 4 } & Vocal & Lt. hand & Rt. hand & Vocal & Lt. hand & Vocal & Rt. hand \\
Mean & 205.3 & 139.9 & 141.1 & & 206.1 & 149.5 & 212.5 & 159.9 \\
S.D. & 26.0 & 9.1 & 11.3 & & 29.3 & 14.3 & 29.0 & I9.0 \\
\hline
\end{tabular}

\section{Discussion}

The present results indicate that $\mathrm{RT}$ s of the simultaneous tasks are longer than those of the single tasks, and that slowing of RTs in the simultaneous tasks are larger on the manual than vocal response, particularly on the right side. This asymmetry of the interference effect was not observed in the bilateral simultaneous 
movements of arms and hands (Taniguchi et al. 1977; Nakamura et al. 1977). Thus, the asymmetry of the interference effect would be elicited only in the case of simultaneous tasks of the manual and vocal performance.

Kinsbourne and Hicks (1978) have suggested that, when the voice and one hand are concurrently active, there will be greater interference between the voice and the right hand than between the voice and the left hand, assuming that the vocal response should be controlled predominantly by the left hemisphere.

However, our data have shown that the vocal response was little affected by combined performance of either side of the hand. Karp et al. (1971) reported that the verbal response, saying 'Now' to the electrical cutaneous stimuli, was not dependent on the side of the cerebral lesion. In our observations, patients with aphasia could perform the present tasks as normal subjects did. It is well known that vocalization occurs with equal frequency from stimulation of the dominant and the non-dominant hemispheres (Penfield and Rasmussen 1952). The vocal response in the present experiment seems to be performed by either of the two hemispheres with nearly equal potentiality. Therefore, compared with the manual response, the vocal response would be less affected in the stimultaneous tasks.

If the vocal response is executed by both hemispheres, a greater interference of vocal to the right than to the left manual response should be attributed to a different mode of processing in the left and right hemispheres, for example, a serial and a parallel processing characteristic to the respective hemispheres (Cohen 1973).

\section{References}

1) Bowers, D., Heilman, K.M., Satz, P. \& Altman, A. (1978) Simultaneous performance on verbal, nonverbal and motor tasks by right-handed adults. Cortex, 14, 540-556.

2) Cohen, G. (1973) Hemispheric differences in serial versus parallel processing. $J$. exp. Psychol., 97, 349-356.

3) Hicks, R.E. (1975) Intrahemispheric response competition between vocal and unimanual performance in normal adult human males. J. comp. Physiol. Psychol., 89, 50-60.

4) Karp, E., Belmont, I. \& Birch, H.G. (1971) Delayed sensory-motor processing following cerebral damage. Cortex, 7, 419-425.

5) Kinsbourne, M. \& Cook, J. (1971) Generalized and lateralized effects of concurrent verbalization on a unimanual skill. Quart. J. exp. Psychol., 23, 341-345.

6) Kinsbourne, M. \& Hicks, R.E. (1978) Functional cerebral space: A model for overflow, transfer and interference effects in human performance. In: Attention and Performance, vol. 7, edited by J. Requin, Lawrence Erlbaum, New Jersey, 345-362.

7) Lomas, J. \& Kimura, D. (1976) Intrahemispheric interaction between speaking and sequential manual activity. Neuropsychol., 14, 23-33.

8) Low, D.W. \& Rebert, C.S. (1978) Sex differences in cognitive/motor overload in reaction time tasks. Neuropsychol., 16, 611-616.

9) McFarland, K.A. \& Ashton, R. (1975) The lateralized effects of concurrent cognitive activity on a unimanual skill. Cortex, 11, 283-290.

10) McFarland, K.A. \& Ashton, R. (1978) The influence of brain lateralization of function on a manual skill. Cortex, 14, 102-111.

11) Nakamura, R., Sajiki, N. \& Taniguchi, R. (1977) Reaction time in bimanual simultaneous motions. J. Human Ergol., 6, 69-73. 
12) Penfield, W. \& Rasmussen, T. (1952) The Cerebral Cortex of Man. A Clinical Study of Localization of Function. Macmillan, New York.

13) Rizzolatti, G., Bertoloni, G. \& Buchtel, H.A. (1979) Interference of concomitant motor and verbal tasks on simple reaction time: A hemispheric difference. Neuropsychol., 17, 323-330.

14) Taniguchi, R., Nakamura, R. \& Oshima, Y. (1977) Reaction time in simultaneous motions. Percept. Motor Skills, 44, 709-710. 